PROCEEDINGS OF THE

AMERICAN MATHEMATICAL SOCIETY

Volume 127, Number 12, Pages 3729-3731

S 0002-9939(99)05135-7

Article electronically published on August 3, 1999

\title{
OPENNESS AND MONOTONEITY OF INDUCED MAPPINGS
}

\author{
WŁODZIMIERZ J. CHARATONIK
}

(Communicated by Alan Dow)

\begin{abstract}
It is shown that for locally connected continuum $X$ if the induced mapping $C(f): C(X) \rightarrow C(Y)$ is open, then $f$ is monotone. As a corollary it follows that if the continuum $X$ is hereditarily locally connected and $C(f)$ is open, then $f$ is a homeomorphism. An example is given to show that local connectedness is essential in the result.
\end{abstract}

All spaces considered in this paper are assumed to be metric. A mapping means a continuous function. We denote by $\mathbb{N}$ the set of all positive integers, and by $\mathbb{C}$ the complex plane. Given a space $S$, a point $c \in S$ and a number $\varepsilon>0$, we denote by $B_{S}(c, \varepsilon)$ the open ball in $S$ with center $c$ and radius $\varepsilon$.

A continuum means a compact connected space. Given a continuum $X$ with a metric $d$, we let $2^{X}$ denote the hyperspace of all nonempty closed subsets of $X$ equipped with the Hausdorff metric $H$ defined by

$$
H(A, B)=\max \{\sup \{d(a, B): a \in A\}, \sup \{d(b, A): b \in B\}\}
$$

(see, e.g., [5, (0.1), p. 1 and $(0.12)$, p. 10]). Further, we denote by $C(X)$ the hyperspace of all subcontinua of $X$, i.e., of all connected elements of $2^{X}$, and by $F_{1}(X)$ the hyperspace of singletons. The reader is referred to Nadler's book [5] for needed information on the structure of hyperspaces.

Given a mapping $f: X \rightarrow Y$ between continua $X$ and $Y$, we consider mappings (called the induced ones)

$$
2^{f}: 2^{X} \rightarrow 2^{Y} \quad \text { and } \quad C(f): C(X) \rightarrow C(Y)
$$

defined by

$2^{f}(A)=f(A)$ for every $A \in 2^{X}$ and $C(f)(A)=f(A)$ for every $A \in C(X)$.

A mapping between continua is said to be:

- open provided the image of an open subset of the domain is open in the range;

- monotone provided the point-inverses are connected;

- light provided the point-inverses are zero-dimensional.

The following theorem is the main result of this paper.

1. Theorem. Let a continuum $X$ be locally connected, and a mapping $f: X \rightarrow$ $Y$ be such that the induced mapping $C(f): C(X) \rightarrow C(Y)$ is open. Then $f$ is monotone.

Received by the editors June 16, 1997 and, in revised form, January 4, 1998.

1991 Mathematics Subject Classification. Primary 54B20, 54F15, 54E40.

Key words and phrases. Continuum, hyperspace, induced mapping, open, monotone.

(C)1999 American Mathematical Society 
Proof. Assume $f$ satisfies the assumptions of the theorem and that it is not monotone. Let $p$ and $q$ be two points of $X$ such that $f(p)=f(q)$ that belong to different components of $f^{-1}(f(p))$. By continuity of $f$ there is a positive $\varepsilon$ such that for every continuum $L \subset Y$ such that $f(p) \in L$ and $H(L,\{f(p)\})<\varepsilon$ the components of $f^{-1}(L)$ containing $p$ and $q$ respectively are distinct. By local connectedness of $Y$ there is a continuum $V$ such that $f(p) \in \operatorname{int} V$ and $H(V,\{f(p)\})<\varepsilon$, i.e., $V \subset B_{Y}(f(p), \varepsilon)$. Let $U_{p}$ and $U_{q}$ be components of $f^{-1}(V)$ containing $p$ and $q$ respectively. Since in locally connected continua components of open sets are open $\left[4, \S 49\right.$, II, Theorem 4, p. 230], we conclude that $p \in \operatorname{int} U_{p}$ and $q \in \operatorname{int} U_{q}$. Let $\delta>0$ be such that $B_{X}(p, \delta) \subset U_{p}$ and $B_{X}(q, \delta) \subset U_{q}$.

Let $\mathcal{B}$ be an order $\operatorname{arc}$ in $C(Y)$ from $\{f(p)\}$ to $Y$ through $V$. Define $\mathcal{A}$ as a subset of $\mathcal{B}$ composed of all elements $L \in \mathcal{B}$ such that the component of $f^{-1}(L)$ containing $p$ is distinct from the component of $f^{-1}(L)$ containing $q$. Note that $V \in \mathcal{A}$ and that if $L, L^{\prime} \in \mathcal{B}, L \in \mathcal{A}$ and $L^{\prime} \subset L$, then $L^{\prime} \in \mathcal{A}$. Thus $\mathcal{A}$ is a connected subset of $\mathcal{B}$ containing $\{f(p)\}$ and $V$. Since $\mathcal{B} \backslash \mathcal{A}$ is closed, we see that $\mathcal{A}$ is an open subset of $\mathcal{B}$. Let $Q=\sup \mathcal{A}=\inf (\mathcal{B} \backslash \mathcal{A})$. Then $Q \in \operatorname{cl} \mathcal{A} \backslash \mathcal{A}$. Denote by $P$ the component of $f^{-1}(Q)$ containing both $p$ and $q$. Openness of $C(f)$ implies that $f$ is open (see $[3$, Theorem 4.3, p. 243]; compare also $[2$, Theorem 3.2]), so $f(P)=Q[6,(7.5)$, p. 148]. We will show that $C(f)\left(B_{C(X)}(P, \delta)\right)$ is not open in $C(Y)$. So, assume the contrary. Then there is a continuum $K \in B_{C(X)}(P, \delta)$ with $f(K) \in \mathcal{A}$. Since $p, q \in P$ and $H(P, K)<\delta$, we have $K \cap U_{p} \neq \emptyset \neq K \cap U_{q}$. Then $U_{p} \cup K \cup U_{q}$ is a continuum containing both $p$ and $q$, whose image $f\left(U_{p} \cup K \cup U_{q}\right)=f(K)$ is in $\mathcal{A}$, contrary to the definition of $\mathcal{A}$. The proof is finished.

2. Corollary. Let a continuum $X$ be hereditarily locally connected, and a mapping $f: X \rightarrow Y$ be such that the induced mapping $C(f): C(X) \rightarrow C(Y)$ is open. Then $f$ is a homeomorphism.

Proof. It is enough to show that monotone open mappings on hereditarily locally connected continua are homeomorphisms. Assume the contrary, and let $y \in Y$ be such that $f^{-1}(y)$ is a nondegenerate continuum in $X$. Let $\left\{y_{n}\right\}$ be an arbitrary sequence converging to $y$. Then continua $f^{-1}\left(y_{n}\right)$ tend to $f^{-1}(y)$, so $f^{-1}(y)$ is a nondegenerate continuum of convergence, contrary to hereditary local connectedness of $X$.

3. Example. There are a continuum $X$ and a mapping $f: X \rightarrow X$ such that $C(f): C(X) \rightarrow C(X)$ is light and open, but not monotone.

Proof. Let $S=\{z \in \mathbb{C}:|z|=1\}$ be the unit circle. For $n \in \mathbb{N}$ put $X_{n}=S$, and let $\varphi_{n}: X_{n+1} \rightarrow X_{n}$ be defined by $\varphi_{n}(z)=z^{3}$. Then $X=\lim _{2}\left(X_{n}, \varphi_{n}\right)$ is the triodic solenoid. Define $f: X \rightarrow X$ by $f\left(\left\{z_{1}, z_{2}, \ldots\right\}\right)=\left\{z_{1}^{2}, z_{2}^{2}, \ldots\right\}$, and note that $f$ is well-defined. It has been proved in [1, Example 4.5] that the restriction $C(f) \mid(C(X) \backslash\{X\})$ is two-to-one and $C(f)^{-1}(X)$ is a singleton. Thus $C(f)$ is light and it is not a homeomorphism. We will prove that $C(f)$ is open. To this aim it is enough to show that the mapping is interior at each point of its domain $[6, \mathrm{p}$. 149], i.e., that for each $P \in C(X)$ and for each open neighborhood $\mathcal{U}$ of $P$ in $C(X)$ we have $C(f)(P) \in \operatorname{int} C(f)(\mathcal{U})$. For each $n \in \mathbb{N}$ let $f_{n}: X_{n} \rightarrow X_{n}$ be defined by $f_{n}(z)=z^{2}$ (and thus $f=\lim _{n} f_{n}$ ), and let $\pi_{n}: X \rightarrow X_{n}$ be the projection. Let $P \in$ $C(X)$ be a proper subcontinuum of $X$. Then there exists an index $n \in \mathbb{N}$ such that $\pi_{n-1}(P)$ is a proper subcontinuum of $X_{n-1}$, so $\pi_{n}(P)$ is an arc of length less than $2 \pi / 3$. Let $U_{n}$ be an open arc in $X_{n}$ containing $\pi_{n}(P)$ and having its length still less 
than $2 \pi / 3$. Then the set $\mathcal{V}=\left\{A \in C(X): \pi_{n}(A) \in U_{n}\right\}$ is an open neighborhood of $P$ in $X$ such that the restriction $C(f) \mid \mathcal{V}: \mathcal{V} \rightarrow C(f)(\mathcal{V})$ is a homeomorphism onto the open set $C(f)(\mathcal{U})=\left\{A \in C(X): \pi_{n}(A) \in f_{n}\left(U_{n}\right)\right\}$ containing $C(f)(P)$. So interiority of $C(f)$ at $P$ is shown in the case $P \neq X$. To prove that $C(f)$ is interior at $X$ consider, for $n \in \mathbb{N}$, the sets $\mathcal{V}_{n}=\left\{A \in C(X): \pi_{n}(A)=X_{n}\right\}$ and note that the family $\left\{\mathcal{V}_{n}: n \in \mathbb{N}\right\}$ is a local base of (closed) neighborhoods of $X$ on $C(X)$. So, it is enough to prove that $C(f)\left(\mathcal{V}_{n}\right) \supset \mathcal{V}_{n+1}$. To this end take $A \in \mathcal{V}_{n+1}$, and let $B \in X$ be such that $f(B)=A$. Since

$$
f_{n+1}\left(\pi_{n+1}(B)\right)=\pi_{n+1}(f(B))=\pi_{n+1}(A)=X_{n+1},
$$

we see that $\pi_{n+1}(B)$ is an arc in $X_{n+1}$ of length at least $\pi$. Thus $\pi_{n}(B)=$ $\varphi_{n}\left(\pi_{n+1}(B)\right)=X_{n}$, i.e., $B \in \mathcal{V}_{n}$, whence it follows that $A=f(B) \in C(f)\left(\mathcal{V}_{n}\right)$. The proof is then complete.

In connection with Theorem 1 and Example 3 it would be interesting to know if a stronger result is true, namely whether or not the conclusion of Theorem 1 can be deduced from local connectedness of $Y$ only (without assuming local connectedness of $X)$. In other words we have the following question.

4. Question. Can the assumption of local connectedness of the domain continuum $X$ be relaxed to that of the range continuum $Y$ in Theorem 1 ?

\section{REFERENCES}

1. J. J. Charatonik and W. J. Charatonik, Lightness of induced mappings, Tsukuba J. Math. 22 (1998), 179-192. CMP 98:16

2. H. Hosokawa, Induced mappings between hyperspaces II, Bull. Tokyo Gakugei Univ. (4) 44 (1992), 1-7. MR 94a:54036

3. H. Hosokawa, Induced mappings on hyperspaces, Tsukuba J. Math. 21 (1997), 239-250. MR 98f:54011

4. K. Kuratowski, Topology, vol. 2, Academic Press and PWN, New York, London and Warszawa, 1968. MR 41:4467

5. S. B. Nadler, Jr., Hyperspaces of sets, M. Dekker, 1978. MR 58:18330

6. G. T. Whyburn, Analytic topology, Amer. Math. Soc. Colloq. Publ. 28, Providence, 1942, reprinted with corrections 1971. MR 32:425

Mathematical Institute, University of Wroceaw, Pl. Grunwaldzki 2/4, 50-384 WrocEAW, POLAND

E-mail address: wjcharat@hera.math.uni.wroc.pl

Departamento de Matemáticas, Facultad de Ciencias, Universidad Nacional Autónoma de México, Circuito Exterior, Ciudad Universitaria, 04510 México, D. F., México

E-mail address: wjcharat@lya.fciencias.unam.mx 